\title{
Spindle cell hemangioma of nasal passage and ethmoidal sinus in a 4-month old infant
}

\author{
Tugba Tastemel Ozturk, M.D. ${ }^{a}$, Ahmet E. Suslu, Professor ${ }^{b}$, Altan Kavuncuoglu, M.D. ', Ekim Gumeler, M.D. ${ }^{d}$, \\ Kemal Kosemehmetoglu, Associated Professor ${ }^{c}$ and Bilgehan Yalcin, Professor ${ }^{e}$
}

\begin{abstract}
Spindle cell hemangioma (SCH) is a benign unusual vascular neoplasm. It does not have gender predilection and can occur at all ages. The disease affects dermis and subcutis of distal extremities predominantly; head and neck involvement is very rare, paranasal sinus involvement has not been reported before. Herein we present a 4-month-old infant with nasal obstruction since two weeks of age due to a mass in ethmoid sinus obliterating the nasal passage. After the histopathological diagnosis of $\mathrm{SCH}$, the tumor was partially resected. In the sixth month follow-up, there was minimal regression of residual lesions. In the imaging studies performed 30 months after the surgery, the residual mass was found to be disappeared. SCH is not frequent in the head and neck, and presentation of some patients may not suggest the diagnosis. Histopathology is important for differential diagnosis and to orientate treatment. Awareness of $\mathrm{SCH}$ may increase the reported cases.

Key words: pediatrics, head and neck neoplasms, paranasal sinuses, spindle cell hemangioma.
\end{abstract}

http: / / dx.doi.org/10.5546/ aap.2021.eng.e36

To cite: Tastemel Ozturk T, Suslu AE, Kavuncuoglu A, Gumeler E, et al. Spindle cell hemangioma of nasal passage and ethmoidal sinus in a 4-month old infant. Arch Argent Pediatr 2021;119(1):e36-e40.

a. Department of Pediatrics.

b. Department of Otolaryngology.

c. Department of Pathology.

d. Department of Radiology.

e. Division of Pediatric Oncology, Department of Pediatrics.

Hacettepe University Faculty of Medicine, Ankara, Turkey.

E-mail address:

Tugba Tastemel Ozturk, M.D.: t_tastemel@hotmail.com

Funding: None.

Conflict of interest: None.

Received: 2-26-2020

Accepted: 8-28-2020

\section{INTRODUCTION}

Spindle cell hemangioma ( $\mathrm{SCH}$ ) is an unusual vascular lesion described in 1986 by Weiss and Enzinger as spindle cell hemangioendothelioma resembling cavernous hemangioma and Kaposi sarcoma. ${ }^{1}$ This lesion was defined as a lowgrade angiosarcoma initially, but owing to lack of metastasis and excellent prognosis, it was considered as a benign vascular neoplasm despite the high rate of local recurrences, and termed as $\mathrm{SCH}$ in $1996 .^{1,2}$ Spindle cell hemangioma does not have gender predilection and can occur at all ages. It affects dermis and subcutis of distal extremities predominantly. ${ }^{2}$ Head and neck region involvement is very rare. To our knowledge, 11 cases of SCH have been reported in the head and neck region so far, and paranasal sinus involvement has not been reported before. ${ }^{3}$ Herein we present a 4-month-old baby with nasal obstruction due to a $\mathrm{SCH}$ mass in ethmoid sinus obliterating the nasal passage.

\section{CASE REPORT}

A 4-month-old baby boy was admitted to the otorhinolaryngology clinic with the complaint of nasal obstruction. His parents said he had slight swelling on his left eyelid and a nasal obstruction from the second week after birth. They had noticed a mass in the nose in the second month after birth. The patient was taken to another hospital where he underwent a surgical excision and most of the mass was removed, which was found to obstruct the left nasal passage.

We didn't have any information concerning the initial histopathology of the resected tissues. Considering the prominent vascular nature of the lesion, oral propranolol had been started at the previous hospital. The patient was admitted to our hospital when he experienced nasal obstruction again two months after surgery. He was assessed in the Otorhinolaryngology Department, and an incisional biopsy of the mass in the left nasal passage was performed. Histopathological examination revealed a spindle cell tumor with solid and cleft-like vascular spaces beneath the respiratory epithelium (Figure 1A). On 
high magnification, the tumor consisted of bland epithelioid endothelial cells that did not display cytological atypia and contained intracytoplasmic vacuoles. Erythrocyte extravasation was present (Figure 1B). The tumor was also accompanied by hyalinized papillary areas lined by single layer endothelial cells consistent with papillary endothelial hyperplasia (Figure 1C). Immunohistochemical studies showed that the tumor cells were positive with CD31 supporting its vascular origin (Figure 1D). The tumor was negative with HHV-8, D2-40, GLUT1, and desmin, respectively helping differentiation from Kaposi sarcoma, Kaposiform hemangioendothelioma, infantile hemangioma, and rhabdomyosarcoma. Based on these findings, the patient was diagnosed with $\mathrm{SCH}$ and papillary endothelial hyperplasia.
Magnetic resonance imaging (MRI) revealed a lobulated mass in the left ethmoid sinus, extending inferiorly to the middle and superior nasal meatus, superiorly to left frontal sinus via frontoethmoidal recesses. The medial and superior orbital extraconal area was infiltrated through lamina papyracea and inferior wall of the frontal sinus. Scalloping of the superior wall of the frontal sinus was present. The mass was hyperintense on T2 weighted images and hypointense on T1 weighted images, enhanced vividly but heterogeneously after gadoliniumbased contrast media injection on T1 weighted images (Figure 2). Vascular flow voids were present in the T2 weighted images and postcontrast $\mathrm{T} 1$ weighted images.

The patient was under follow-up
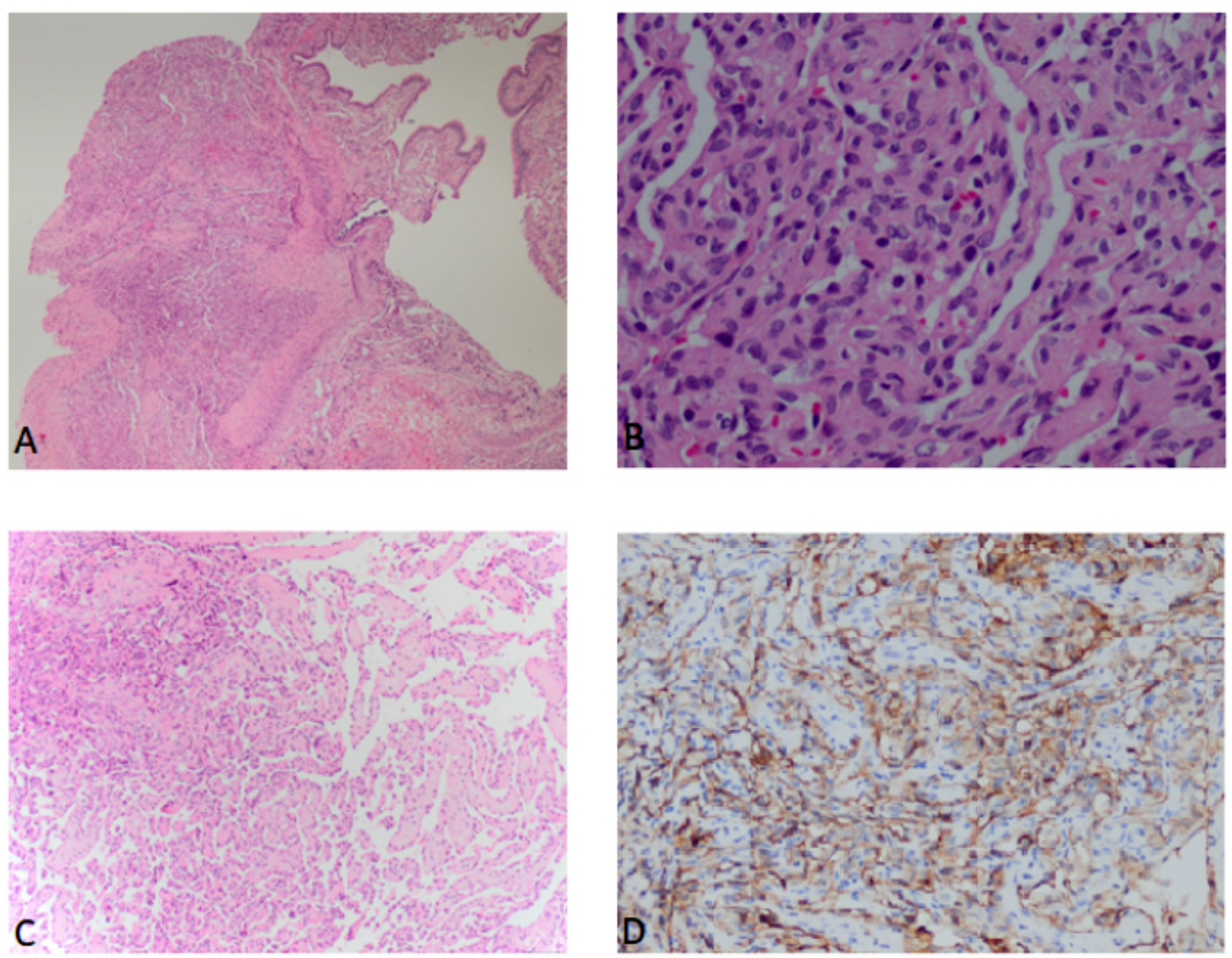

(A) The tumor is seen beneath the respiratory epithelium and contains vascular clefting (hematoxylin and eosin staining, $x 4$ ).

(B) Endothelial cell proliferation with intracytoplasmic vacuoles and accompanying erythrocyte extravasation (hematoxylin and eosin staining, $x 40)$.

(C) Areas of papillary endothelial hyperplasia (hematoxylin and eosin staining, x10).

(D) The tumor cells were positive with CD31 immunohistochemically. 
conservatively since surgery was deemed risky and oral propranolol treatment was continued. But, his nasal obstruction complaint didn't improve, and he started to have frequent nosebleeds. Propranolol was discontinued when he was 8-month-old, and surgical resection was decided. Under general anesthesia, the mass was removed endoscopically. The mass was fragile and vascular and occupied the ethmoid region. The anterior skull base and orbit were explored, the lamina papyracea was defective. The mass was dissected from periorbitis and skull base under endoscopic vision. It was removed in four pieces, the largest one was $3 \times 2.5 \times 1.3 \mathrm{~cm}$, and the specimen was macroscopically brown and irregular. Histopathological examination revealed spindle cell hemangioma similar to the initial biopsy result.

An MRI performed three months after surgery revealed a $1.5 \times 1.7 \times 1 \mathrm{~cm}$ residual lesion in the left frontal sinus and frontoethmoidal recess, with the same aforementioned imaging features (Figure 2). In the sixth month follow-up, there was minimal regression of residual lesions. There were no significant complaints during the follow-up, and in the imaging studies performed 30 months after surgery, it was found that the residual mass had disappeared.

Informed consent was received from the family.

\section{DISCUSSION}

Spindle cell hemangioma is an uncommon benign lesion that has a predilection to extremities and may present as solitary or multifocal, painless or painful mass. ${ }^{2,4}$ Spindle cell hemangioma commonly appears as a nodule on extremities. ${ }^{2}$ Although 11 cases of $\mathrm{SCH}$ localized in the head and neck region have been reported previously, ${ }^{3}$ our case is interesting and unusual because, to our knowledge, it is the first case with paranasal sinus involvement. While most of the reported cases were asymptomatic, our patient had swelling in the left eyelid, nasal obstruction, and nasal bleeding. Spindle cell hemangioma can be seen at any age, ${ }^{2}$ and our case can be considered congenital because the swelling in the left eyelid had been noticed since birth and nasal obstruction since the second week of age.

While most SCH lesions are smaller than

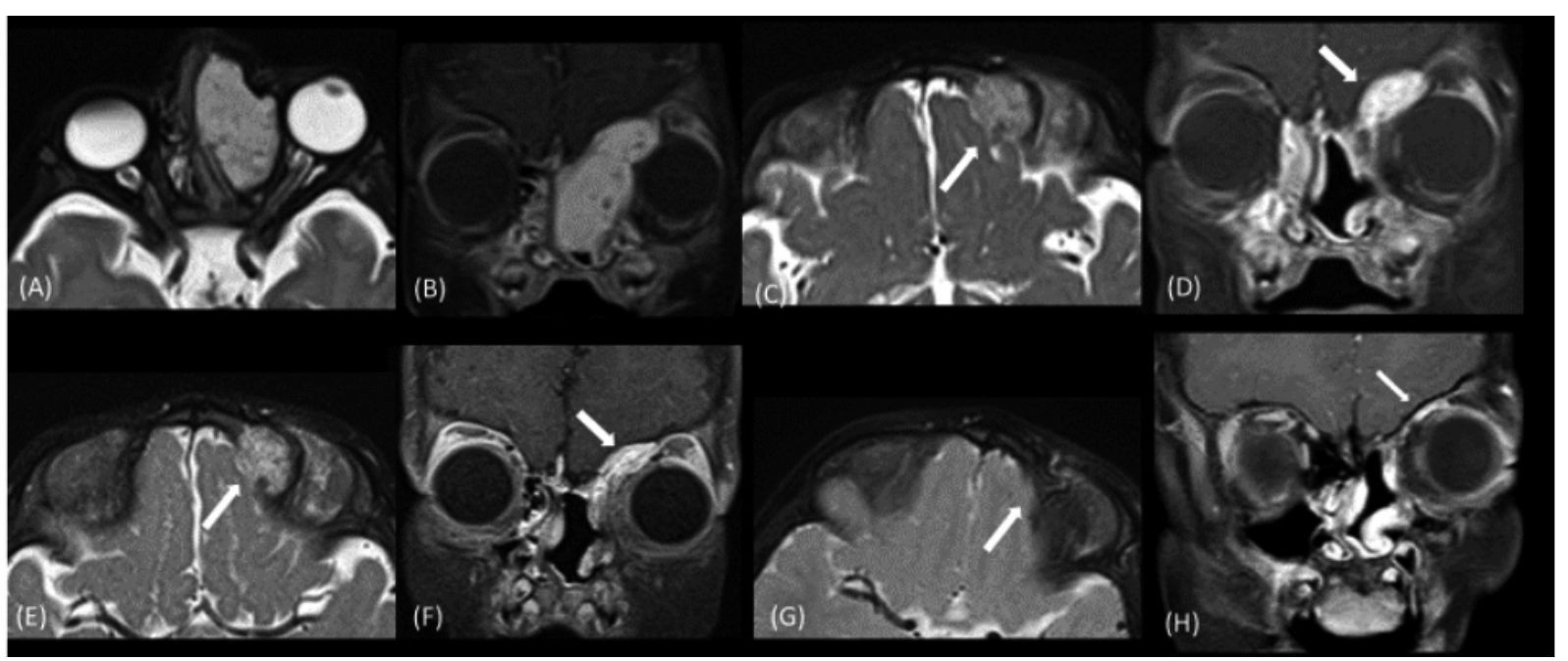

Axial fat-suppressed (FS) T2 weighted image shows a hyperintense mass with scalloping-thinning of lamina paprycea and extending to extraconal orbital fat (A). Postcontrast FS coronal T1 weighted image reveals intense but heterogeneous enhancement of the mass (B). Axial T2 weighted image (C) and postcontrast FS coronal T1 weighted image (D), three months after surgery, show residual mass at frontoethmoidal recess (arrows). The residual mass shows gradual decrease in size six months after the surgery (arrows), shown on axial T2 weighted image (E) and postcontrast FS coronal T1 weighted image (F). On the latest follow-up MRI, 30 months after the surgery, axial T2 weighted image (G) and postcontrast FS coronal T1 weighted image $(\mathrm{H})$ show no sign of active disease (arrows). 
$2 \mathrm{~cm},{ }^{5}$ in our case, it was remarkable that the surgically removed material was much bigger.

Histopathological examination of spindle cell hemangioma consists of cavernous vascular spaces that may have thrombus and lined by endothelial cells and solid spindle cell areas. Spindle cell areas also have epithelioid endothelial cells with cytoplasmic vacuoles. Nuclear atypia is generally low in $\mathrm{SCH}$, and it rarely shows mitotic activity. ${ }^{6}$ But, this case of $\mathrm{SCH}$ also had papillary endothelial hyperplasia areas, which was rarely reported in the literature. ${ }^{5,7,8}$ In the immunohistochemical examination of $\mathrm{SCH}$, capillary and cavernous vessels are positive for CD31, and spindle cell area is positive for smooth muscle actin. ${ }^{9}$ Although the lesion was thought to originate from the blood vessels, a recent study showed endothelial cells of $\mathrm{SCH}$ are positive for PROX-1 (expressed in lymphatic proliferation) and suggested $\mathrm{SCH}$ as a lymphatic malformation. ${ }^{10}$ Recently, somatic mosaic mutations in IDH1 (isocitrate dehydrogenase) and IDH2 were described in $\mathrm{SCH}$ and not in other vascular lesions. Kurek et al, showed $71 \%$ of SCH had IDH1/IDH2 mutation. ${ }^{11}$ Another study revealed that $16 / 17$ of $S C H$ cases had IDH1/IDH2 mutation, and these mutations were not found in other vascular anomalies involving lymphatic malformation. So, it was concluded that this mutation is highly specific for $\mathrm{SCH}$ and can be used for diagnosis. ${ }^{12}$

Multiple lesions of SCH may be associated with Maffucci's syndrome, Klippel-Trenaunay syndrome, congenital lymphedema, and varicose veins. ${ }^{2,7}$ Our patient had no findings of these syndromes.

Although SCH is a benign lesion, recurrences up to $58 \%$, have been reported. ${ }^{2}$ More than half of the lesions are localized partially or totally intravascular, and local recurrences occur through these vessels in the region where the primary lesion is located. The overall prognosis is excellent with total surgical excision. No metastases and no deaths due to $\mathrm{SCH}$ have been reported. ${ }^{2}$ Cases with spontaneous regression have also been reported. ${ }^{7}$ Endoscopic transnasal surgery was performed to our patient considering the advantages of low morbidity, no need for an incision, and providing detailed vision. However, total excision could not be performed due to the localization of the mass. Fortunately, in our patient, the residual mass regressed spontaneously and disappeared. Also, it was reported that a 10-year-old girl with $\mathrm{SCH}$ nodules on the ankle and foot had been successfully treated with sclerotherapy. ${ }^{13}$ Radiotherapy is not recommended because a case with malignant transformation and lymph node metastasis has been reported after radiotherapy. ${ }^{1}$ So, we planned to follow the patient closely in terms of recurrence after spontaneous regression.

Spindle cell hemangioma is not a frequent tumor in the head and neck region, and presenting complaints may not suggest the diagnosis, as in our patient. Since this entity is less well known and has clinical potential to be misdiagnosed as hemangiomas or other vascular lesions, the histopathological diagnosis of such lesions has great importance for treatment planning. Awareness of this diagnosis may increase the reported cases.

\section{REFERENCES}

1. WeissSW,EnzingerFM.Spindlecellhemangioendothelioma. A low-grade angiosarcoma resembling a cavernous hemangioma and Kaposi's sarcoma. Am J Surg Pathol.1986; 10(8):521-30.

2. Perkins P, Weiss SW. Spindle cell hemangioendothelioma. An analysis of 78 cases with reassessment of its pathogenesis and biologic behavior. Am J Surg Pathol. 1996; 20(10):1196204.

3. French KE, Felstead AM, Haacke N, Theaker J, et al. Spindle cell haemangioma of the tongue. J Cutan Pathol. 2016; 43(11):1025-7.

4. Hakozaki M, Tajino T, Watanabe K, Yamada $\mathrm{H}$, et al. Intraosseous spindle cell hemangioma of the calcaneus: a case report and review of the literature. Ann Diagn Pathol. 2012; 16(5):369-73.

5. Tosios KI, Gouveris I, Sklavounou A, Koutlas IG. Spindle cell hemangioma (hemangioendothelioma) of the head and neck: case report of an unusual (or underdiagnosed) tumor. Oral Surg Oral Med Oral Pathol Oral Radiol Endod. 2008; 105(2):216-21.

6. Marušić Z, Billings SD. Histopathology of Spindle Cell Vascular Tumors. Surg Pathol Clin. 2017; 10(2):345-66.

7. Fletcher CD, Beham A, Schmid C. Spindle cell haemangioendothelioma: a clinicopathological and immunohistochemical study indicative of a non-neoplastic lesion. Histopathology. 1991; 18(4):291-301.

8. Tosios K, Koutlas IG, Kapranos N, PapanicolaouSI.Spindlecell hemangioendothelioma of the oral cavity. A case report. J Oral Pathol Med. 1995; 24(8):379-82.

9. TsukamotoS, Honoki K, Shimada K, Fujii H, et al. Periosteal spindle cell hemangioma of the fibula: a case report. Skeletal Radiol. 2013; 42(8):1165-8.

10. Wang L, Gao T, Wang G. Expression of Prox1, D2-40, and WT1 in spindle cell hemangioma. J Cutan Pathol. 2014; 41(5):447-50.

11. Kurek KC, Pansuriya TC, van Ruler MA, van den Akker $\mathrm{B}$, et al. R132C IDH1 mutations are found in spindle cell hemangiomas and not in other vascular tumors or malformations. Am J Pathol. 2013; 182(5):1494-500.

12. Ten Broek RW, Bekers EM, de Leng WWJ, Strengman E, et al. Mutational analysis using Sanger and next generation sequencing in sporadic spindle cell hemangiomas: A study 
e40 / Arch Argent Pediatr 2021;119(1):e36-e40 / Case report

of 19 cases. Genes Chromosomes Cancer. 2017; 56(12):855-60. 13. Kramer D, Downey C, Vargas P, Castro A. Multifocal spindle cell hemangioma: Report of two cases. Indian J Dermatol Venereol Leprol. 2016; 82(1):93-5. 